\title{
MONOLITHIC GEARED-MECHANISMS DRIVEN BY A POLYSILICON SURFACE-MICROMACHINED ON-CHIP ELECTROSTATIC MICROENGINE
}

\author{
Jeffry J. Sniegowski, Sam L. Miller, Glenn F. LaVigne, M. Steven Rodgers and Paul J. McWhorter \\ Integrated Micromechanics, Microsensors, \& CMOS Technology Department \\ Sandia National Laboratories \\ Albuquerque, NM 87185-1080
}

\begin{abstract}
We have previously described a practical micromachined power source: the polysilicon, surface-micromachined, electrostatically-actuated microengine [1]. Here we report on three aspects of implementing the microengine. First, we discuss demonstrations of the first-generation microengine actuating geared micromechanisms including gear trains with elements having dimensions comparable to the drive gear (approximately $50 \mu \mathrm{m})$ and a relatively large $(1600-\mu \mathrm{m}$-diameter) rotating optical shutter element. These configurations span expected operating extremes for the microengine, and address the coupling and loading issues for very-low-aspect-ratio micromechanisms which are common to the design of surfacemicromachined devices. Second, we report on a secondgeneration of designs that utilize improved gear teeth design, a gear speed-reduction unit, and higher force-per-unit-area electrostatic comb drives. The speed-reduction unit produces an overall angular speed reduction of 9.63 and requires dual-level compound gears. Third, we discuss a dynamics model developed to accomplish three objectives: 1) drive inertial loads in a controlled fashion, i.e. specify and achieve a desired timedependent angular position of the drive gear, 2) minimize stress and frictional forces during operation, and 3 ) as a function of time, experimentally determine forces associated with the drive gear, such as load torque being applied to the drive gear due to friction.
\end{abstract}

\section{INTRODUCTION}

Preliminary demonstrations of on-chip powered geared mechanisms have been recently reported $[2,3]$. In the case of on-chip electrostatic actuation of geared mechanisms, the onchip power source must overcome the forces of stiction [4]. The forces generated by on-chip means are limited and relatively small compared to externally applied forces. The result is that initial start-up can be problematic and unreliable. In contrast, start-up and operation issues related to release and in-use stiction are more readily overcome in mechanical systems that are actuated on by external means [5]. We report here the full function operation of various geared mechanisms driven by onchip means and discuss system dynamics which lead to tailored input signals which enhance start-up and operation. Details of the fabrication of these devices are reported elsewhere $[\mathbf{1 , 6 ]}$.

For our initial proof-of-concept demonstration, we chose two principle configurations of the micro-engine and geared micromechanism system. The first, exhibited in Figure 1, consists of the microengine loaded by comparably-sized gears and provides fundamental information on the basic design of the microengine and gears. The second involves the actuation of a relatively large $(1600-\mu \mathrm{m}$-diameter) rotating optical shutter element shown in Figures 2 and 3. Design details for these two configurations are described in a previous report [7]. These configurations span expected operating extremes for the microengine and address the coupling and loading issues for very-low-aspect-ratio micromechanisms which are common to the design of surface-micromachined devices. We then report on a second-generation of designs that utilize improved gear teeth design, a gear speed-reduction unit, and higher force-per-unitarea electrostatic comb drives. Finally, optimization of endurance and of high-speed start-up of large elements through dynamical modeling is discussed.

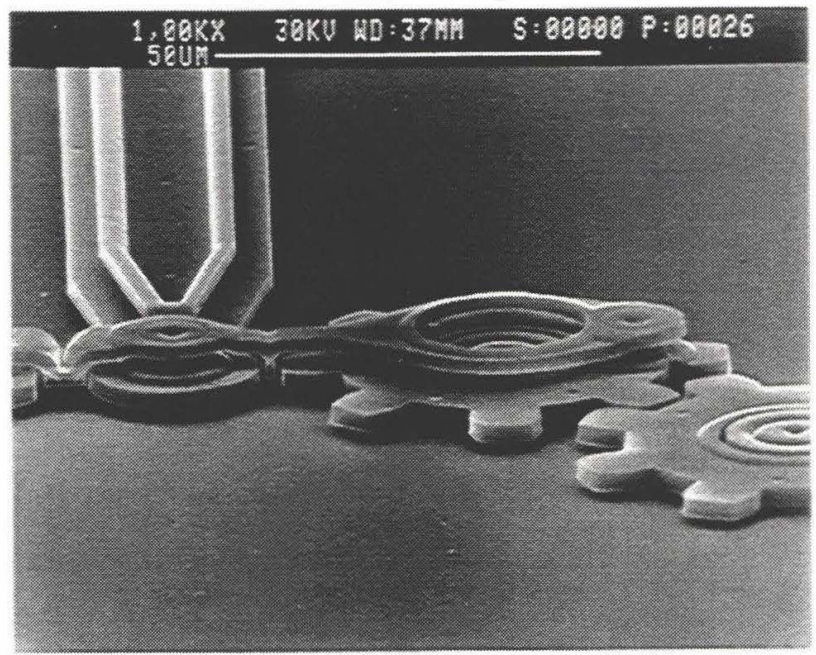

Figure 1. SEM perspective view of the microengine drive gear and comparably sized driven gear. Gear extreme diameter is approximately $60 \mu \mathrm{m}$ and gear thickness is $2.45 \mu \mathrm{m}$.



Figure 2. SEM micrograph overview of the microengine coupled to drive a 1600- $\mu$ m-diameter optical shutter gear. The two linear drives are seen as the two orthogonal electrostatic comb drives in the upper right-hand corner of the micrograph. They are connected by linkages, seen close-up in Figure 3, to the drive gear which is coupled to the driven element by gear teeth.

As a quick review, the principle of operation for the microengine is well described by analogy to two orthogonal pistons connected to a crank shaft, i.e. two linear drive elements connected to a rotary element to produce rotational output. Referring to Figure 2, the linear drive elements, which are electrostatic comb drives [8], are connected by linkages to the rotary drive gear. This drive gear is then coupled directly to 
the large gear through the gear teeth. The close-up in Figure 3 more clearly illustrates the linkages from the linear drives connected to the drive gear.

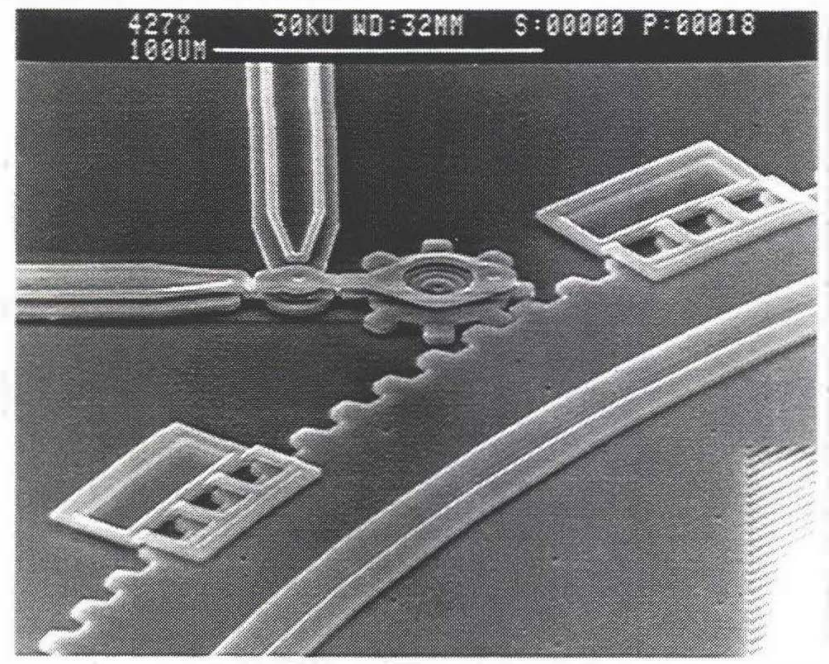

Figure 3. SEM micrograph of the microengine drive gear

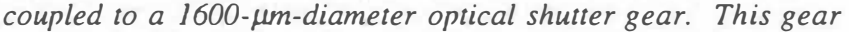
element is 30 times the diameter of the microengine drive gear. Also present in this photo are a set of retainers used to maintain vertical alignment of the sets of gear teeth, which are only 2.45 $\mu m$ thick.

\section{DEVICE DEMONSTRATIONS}

First-Generation Devices - The two devices discussed in this section consist of the microengine driving gears of comparable dimension (Figures 1 and 4), and the microengine driving a $1600-\mu \mathrm{m}$-diameter gear which functions as an optical shutter (Figures 2 and 3).

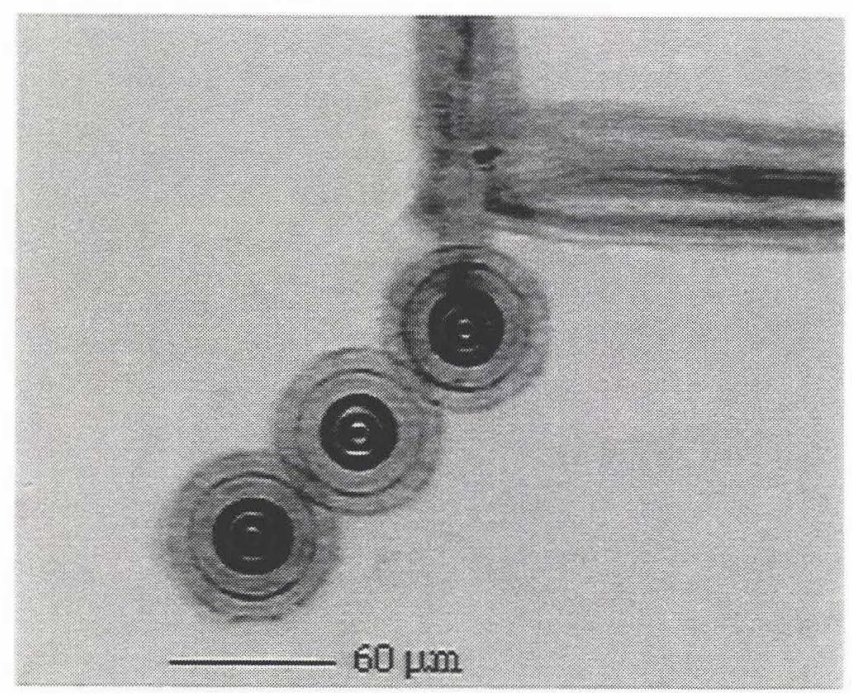

Figure 4. Optical micrograph taken during the operation of the microengine driving two additional gears at 200,000 rpm. Gear extreme diameter is $60 \mu \mathrm{m}$.

The rotation of the drive gear and gear train was achieved by the application of $90 \mathrm{~V}$ square-wave inputs to the combdrive actuators. These tests were performed in ambient air and full rotation of single and multiple gear configurations was demonstrated. During tests conducted using low-frequency square waves, the motion observed is similar to that of a stepper motor. With each step in the applied signals, the drive gear moves to an equilibrium position and remains in that position until the next change of the applied signals. Due to the low inertia of these structures, the movement to the equilibrium positions occurs on a sub-millisecond time scale. Changing the phase relationship between $X$ and $Y$ actuators allows for reversal of the microengine rotation. Several devices were operated at square-wave frequencies ranging from $0.5 \mathrm{~Hz}$ to $3333 \mathrm{~Hz}$ which corresponds to 30 revolutions per minute (rpm) to 200,000 rpm. Figure 4 is an optical micrograph of the drive gear and gear train operating at $200,000 \mathrm{rpm}$.

The dynamical modeling accounts for the inertial load of the drive gears. In the case of the large optical shutter gear, a simple step ramp of the drive signals to the engine produces a maximum speed of $600 \mathrm{rpm}$ for the $1600 \mu \mathrm{m}$-diameter-gear, while a modified ramp of the drive signals as predicted by the modeling has allowed a speed of $4800 \mathrm{rpm}$ to be obtained. These large gear speeds correspond to $\sim 20,000 \mathrm{rpm}$ and $146,000 \mathrm{rpm}$ for the drive gear, respectively. Additional discussion of this topic is covered in the section on dynamical operation model.

Second-Generation Designs - By driving a 1600 $\mu \mathrm{m}$-diameter gear, the first-generation microengines have proven that electrostatically driven assemblies can produce significantly useful work. Further improvements, however, will be required if similar devices are to propel the more complicated micromachines envisioned. We are addressing these needs by developing a new family of microengines that utilize improved gear teeth design [9], elaborate gear-speed-reduction units, and higher force-per-unit-area comb drives. Together these enhancements can significantly increase the torque available for driving mechanical loads.

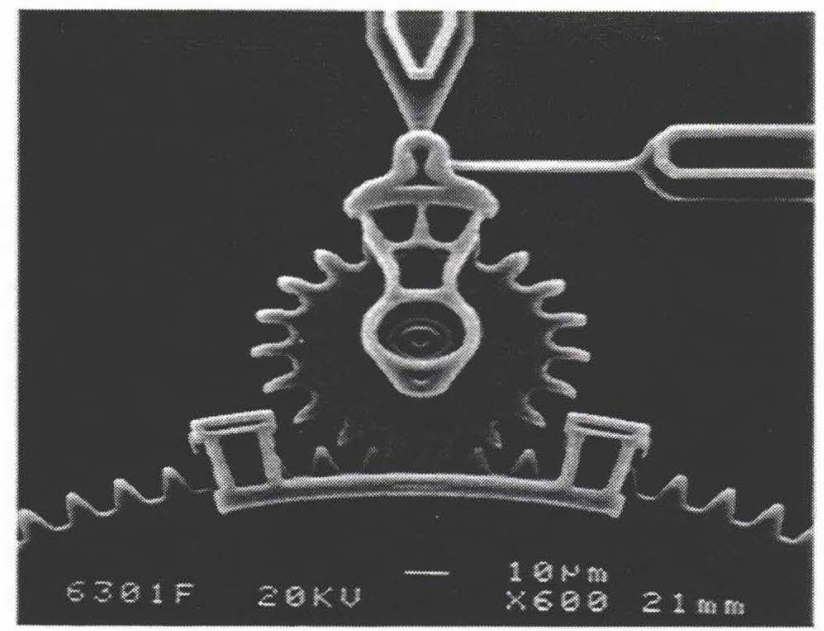

Figure 5. SEM of second-generation tooth design. The drive gear pitch circle radius is increased from $26 \mu \mathrm{m}$ to $38 \mu \mathrm{m}$ to accommodate the deeper tooth design and additional teeth. Note the deeper and multiple tooth engagement relative to the gears in Figure 1 .

Although the first-generation gear tooth shape is involute [7], (refer to Figures 1 and 3) several shortcomings were readily observed. Process constraints on the ratio of the hub-to-gear spacing to the overall gear dimension allowed relative motion of the gears to significantly change the tooth pressure angle and rolling behavior. To reduce this, a primary modification to the design included a larger drive gear diameter to accommodate deeper tooth design and additional teeth for greater engagement and continuous engagement of multiple teeth. The depth was changed from approximately $7 \mu \mathrm{m}$ to $9.5 \mu \mathrm{m}$. A secondary change modified the manner in which the link runs over the gear edge such that the constraint on the tooth tip width was eliminated from the first design. This allowed fabrication of a more ideal tooth shape. Comparison of Figure 5 to Figure 1 
illustrates these changes. These changes eased start-up and produced much smoother operation, particularly at low speed.

A second structure preserved and only slightly modified from the early design is the retainer for out-of-plane gear-tooth alignment. This is the over-clamp at the edge of the large gear in both Figures 3 and 5. A simple evaluation by either including or not including the retainers in the operation of the devices demonstrated that they are highly effective at preventing out-of-plane disengagement of the gears while not appreciably adding to the friction load.

New Second-Generation Devices - The aforementioned design changes led us to consider a gear-speedreduction unit, i.e. a transmission. To construct the unit required design of dual-level compound gears. This was accomplished by full utilization of the three levels of mechanical polysilicon in the basic process. A dual-level gearspeed-reduction unit that drives a rack back and forth is shown in figure 6.

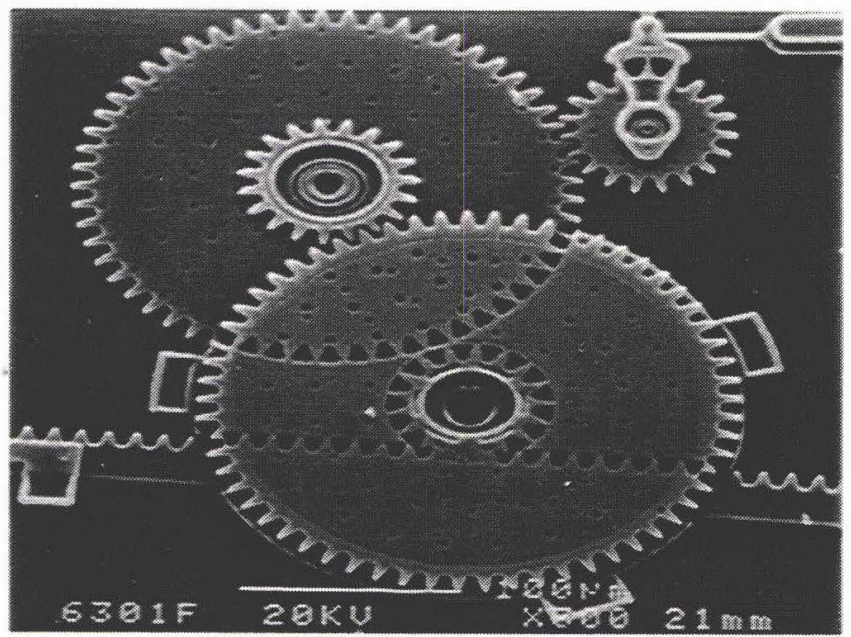

Figure 6 is an SEM of the completed gear-speed-reduction unit and linear rack. The linear speed of the rack is approximately one tenth that of the linear tooth velocity of the drive gear. The rack can be used to drive a folding mirror, for example.

This assembly utilizes a microengine with a 19 tooth pinion [10] to drive two compound gears that have teeth fabricated in level 2 and level 3 polysilicon. The hubs for all the gears are formed from level 1 and level 2 polysilicon. Each compound gear has a center gear that is about $1 / 3$ the diameter of its outer unit. If we take the gears in order of transmission starting with the drive (pinion) gear, the tooth numbers are 19, 57, 19, 61, and 17 respectively. Thus, the linear speed of the rack driven by the speed-reduction unit is $1 / 10.77$ of what it would be if driven directly by the pinion gear. The lower (level 2 poly) components can be seen in Figure 7 . The large flat areas under the final gear are to provide a planar surface for fabrication of the large level 3 gear. The appearance of gear teeth openings in the large top gear in Figure 6 are artifacts that compensate for possible mechanical interferences caused by the conformal nature of the film depositions in the non-planarized process. Such design constraints have been discussed in earlier articles on the microengine $[\mathbf{1 , 6 ]}$. The use of Chemical-Mechanical Polishing for planarization as presented in [11] removes such design constraints.

Bi-directional drive of the rack by the reduction unit has been demonstrated. In addition, removal of the rack from the structure allows continuous high-speed operation. More significant mechanical loads will be coupled to similar microengine driven gear-speed-reduction assemblies in the near future.

Further utilization of all three levels of mechanical polysilicon was made by including a bi-level set of fingers and bi-level support springs on the electrostatic comb drives used to power the rotary drive gear (see Figure 8 ). The net result is analogous to overlaying two comb drives which effectively increases the net output of the linear drives. However, using bilevel springs separated by a two micrometer thick sacrificial oxide essentially triples the effective out-of-plane thickness of the springs. Since the z-direction stiffness is proportional to the thickness cubed, the threefold increase corresponds to approximately a twenty-fold increase in out-of-plane stiffness.

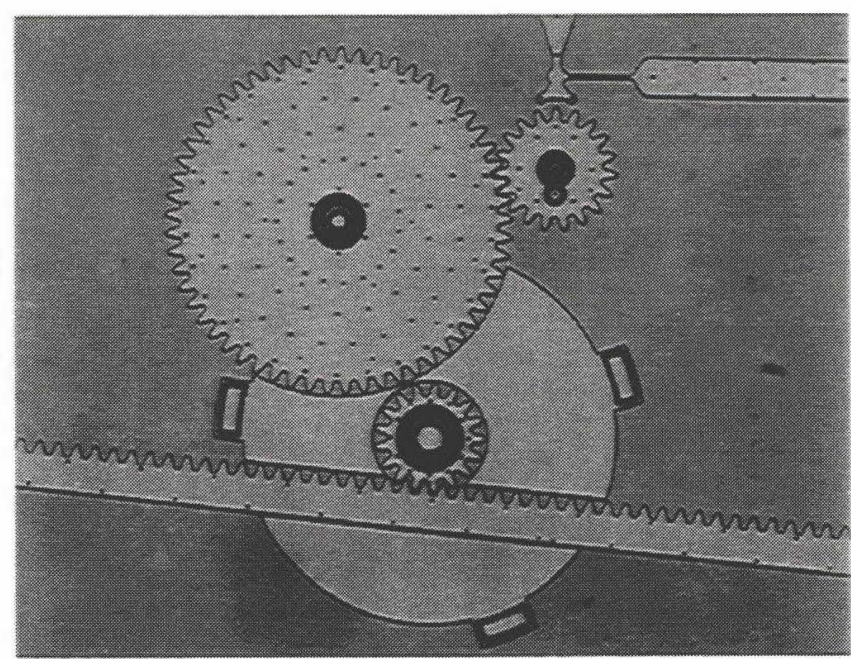

Figure 7. Shown in this micrograph are the three lower level gears and rack in the gear-speed-reduction unit.

This produces a more robust structure that is less susceptible to stiction problems associated with the springs as well as the shuttle. In addition, a more aggressive layout and design has reduced this comb drive's footprint to $2 / 3$ that of its predecessor.

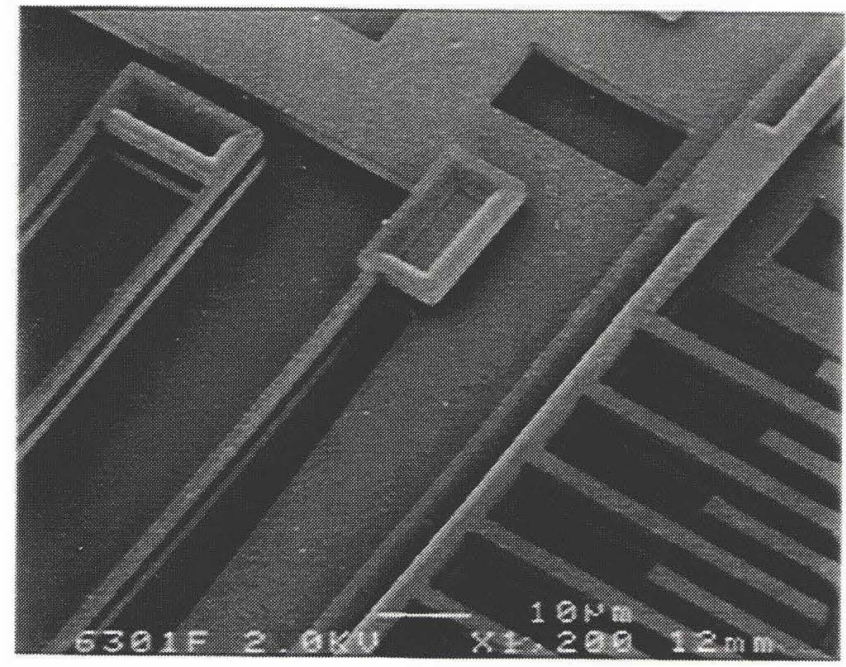

Figure 8. Utilization of multiple levels of mechanical polysilicon is seen in this SEM image of bi-level comb drive fingers and support springs. Each layer is approximately $2 \mu \mathrm{m}$ in thickness. In this case, the bi-level nature of the structures arises from the sacrificial oxide layer that was present between polysilicon layers during fabrication. 
Further Design Issues - The dominant failure mechanism of our second-generation drive is off-axis instability of the electrostatic comb drives leading to a lateral buckling failure under high-speed/high-load operation. The instability has its origin in the basic configuration of a linear comb drive. The linear force generated by the comb structure is derived from the fringing fields at the ends of the comb fingers [1]. These fingers, however, form interdigitated plates of parallel capacitors. Each of these fingers, in its nominal position, sits on an electrostatic potential hill along the crossaxis direction. Therefore, the fingers prefer to collapse into one or the other finger to each side. In the comb drives, supporting springs and/or mechanical stops normally prevent this lateral collapse up to a point. In the case of the current design, the onset of the lateral buckling occurs for an applied voltage of approximately $90 \mathrm{~V}$ under loaded conditions. If there are no connected elements which generate off-axis loads, the onset has not occurred with our latest design at $145 \mathrm{~V}$ which is the current limit of our test set-up.

In order to drive the microengine to higher speeds and larger loads, we have had reasonable success shrinking the spacing to the mechanical stops. In particular, putting a taper on the central shaft of the comb drive in the area of the mechanical stops produces a gap of only a few tenths of a micrometer when the comb is energized. Referring to Figure 9, the gaps on each side of the central shaft are diminished as the central shaft moves between the two stops. Also, we have found that adding any additional lateral stiffness to the entire comb drive is worth the effort. This includes adequate stiffness to the comb fingers. An early change was to increase the lateral width of the fingers from $2 \mu \mathrm{m}$ to $3 \mu \mathrm{m}$ with $2 \mu \mathrm{m}$-finger gaps increased to $3 \mu \mathrm{m}$. Although we have seen improvements in controlling the lateral buckling, we are continuing to modify both the mechanical stops and the lateral stiffness.

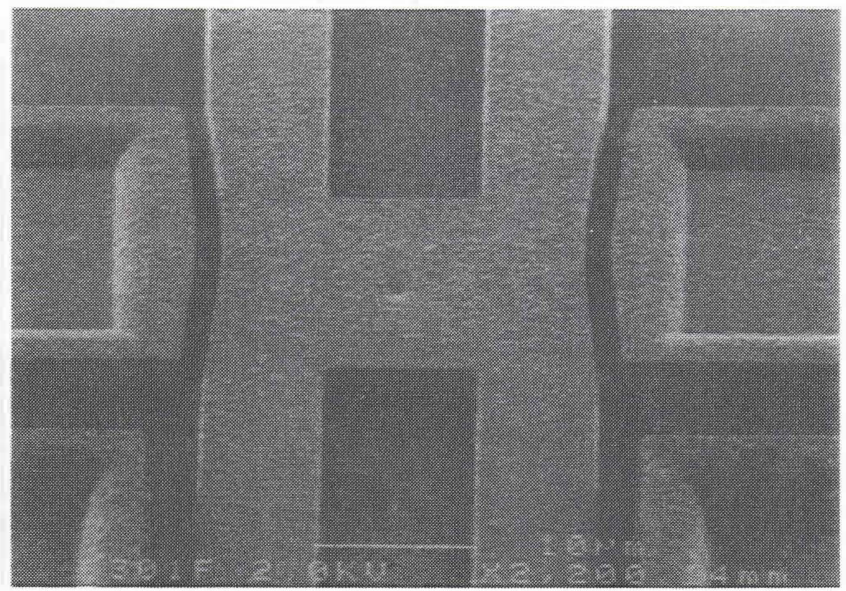

Figure 9. SEM close-up of the central shaft of a linear comb drive. The shaft is the vertical element photo while the stops are seen at both sides. As the shaft moves from this position, it is seen that the spacing to the stops is decreased by the tapers in the shaft.

\section{DYNAMICAL OPERATION MODEL}

The dynamical operation of the microengine is governed by the complex relationship between forces, masses, and the physically constrained geometry associated with its moving elements. Relevant forces include the electrostatic forces originating from the applied comb-drive voltages, restoring forces due to the comb support springs, viscous-damping forces, frictional forces between rubbing surfaces, and inertial forces associated with the linear motion of the comb drives and rotational motion of the gears. Though square-wave or sinewave drive signals can be used to demonstrate functionality and achieve limited operation of the engine, they result in excessive and fluctuating frictional and inertial forces. Such forces can lead to premature failure, and do not result in well-controlled motion of loads being driven by the engine. Specifically, constant angular speed cannot be achieved without the application of specialized drive signals. In addition, rapid acceleration of inertial loads with minimum stress is not possible without properly engineered drive signals.

To enable controlled operation while minimizing parasitic forces, a dynamical model of the engine/load system has been developed and successfully applied. The results are achieved by analytically solving Newton's equations of motion. The model relates the applied voltages, physical device parameters (e.g. force constants, masses and geometry), frictional forces and load torques, and the time-dependent angular position of the drive gear. Application of the model permits the direct experimental determination of the electrostatic, damping,. and spring force constants from functioning engines. The resulting value of Young's modulus is E $\sim 170 \times 10^{9} \mathrm{~N} / \mathrm{m}^{2}$. The measured electrostatic force constant is typically within ten percent of that theoretically expected for the present geometry comb drives [1].

Once the force constants are experimentally determined, the dynamical model is used to experimentally measure the load forces acting on the drive gear. This is possible by measuring the time-dependent angular position of the drive gear, and knowing the applied drive voltages. From a series of different loading conditions, the coefficient of friction between the gear and hub is experimentally measured to be approximately 0.5 .

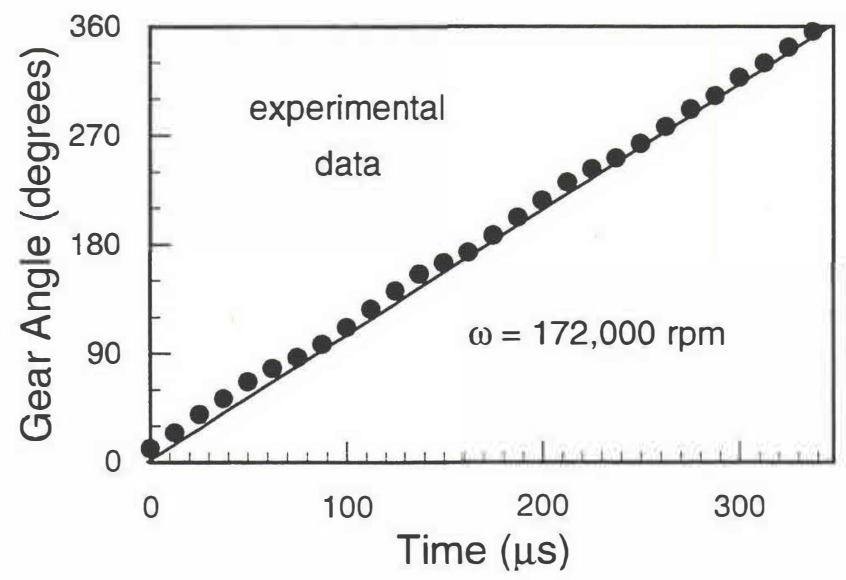

Figure 10. Operation using theoretically computed drive signals results in uniform rotation at speeds in excess of the resonant frequency $(104,000 \mathrm{rpm})$ of the comb drives. Measurements are made using a time-delayed strobe technique.

Controlled operation of the engine can be achieved using the model. To do this, the voltages to be applied to the comb drives are directly computed for a desired angular speed. The experimentally measured angular position of the drive gear as a function of time is shown in Figure 10 for an engine running at a speed of 172,000 revolutions per minute (rpm) operated with drive signals created by the dynamical model. At this speed (nearly twice the resonant frequency of the comb drives), the linear inertial forces of the comb drives significantly impact the dynamics of the engine, and are properly accounted for by the model as evidenced by the approximately constant measured angular speed. The maximum speed of an engine thus far achieved is $300,000 \mathrm{rpm}$. The data shown in Figure 10, and the speed record, were obtained with no load attached to the drive 
gear. At a constant speed, i.e. in the absence of angular acceleration, the rotational inertia of load gears does not impact the engine dynamics.

Rotational inertial effects can be significant during rapid starting and stopping of large gear loads. For example, the $1600-\mu \mathrm{m}$-diameter optical shutter shown in Figure 2 can only be operated up to a maximum speed of $\sim 20,000 \mathrm{rpm}$ (speed of 50$\mu \mathrm{m}$-diameter drive gear) using abruptly starting square-wave drive signals. The speed limitation associated with abrupt starting is caused by the fact that the transfer of electrostatic energy to rotational kinetic energy requires a finite amount of time. In contrast, one can drive the shutter at speeds up to $146,000 \mathrm{rpm}$ by using engineered drive signals created with the dynamical model. Quickly accelerating a load to this speed requires drive signals of complex form that account for both linear and rotational inertial effects. To illustrate, Figure 11 shows drive signals designed to ramp to an engine speed of $140,000 \mathrm{rpm}$ in two revolutions of the drive gear by linearly increasing the instantaneous speed, while at the same time driving the large load gear. For clarity, only the voltages on two of the four comb drives are shown.

Rapid starting and stopping of the engine can result in excessive parasitic forces when improper drive signals are used, even in the absence of a load with rotational inertia. The reason is that the motion of the engine components are physically constrained by the drive gear and linkages. As a result, the hub on which the drive gear rotates provides forces to the accelerating comb drives to compensate for the non-ideal force history provided by the electrostatic comb drives. Such forces can impact the lifetime of the engine. The endurance record achieved thus far for a single engine is $3.2\left(10^{9}\right)$ revolutions while operating at a speed of $146,000 \mathrm{rpm}$. The engine also accrued 66,300 start/stop cycles during the $3.2\left(10^{9}\right)$ revolutions.

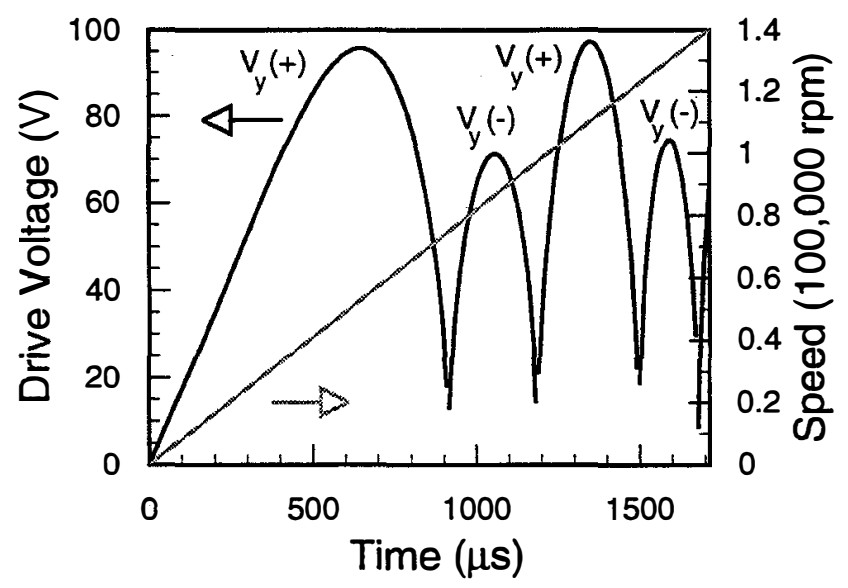

Figure 11. Drive signals to accelerate the 50-um-diameter drive gear to a speed of $140,000 \mathrm{rpm}$ in two revolutions by linearly increasing the speed, while at the same time driving a



\section{CONCLUSION}

In conclusion, this surface-micromachined actuation system can overcome the common start-up and in-use operation difficulties associated with stiction. We are now considering using the microengine as a driver in applications which have requirements from reliable start-up after long dormancy to continuous high-speed operation. The scope of the potential applications have broadened considerably after successful demonstration of improved gear tooth design, a gear-speedreduction unit, and higher force-density comb drives.

\section{ACKNOWLEDGMENT}

We would like to acknowledge the entire staff of the Microelectronics Development Laboratory at Sandia National Laboratories for their efforts in the fabrication of these devices. We also thank Michael Callahan at Sandia National Laboratories for his excellent technical direction regarding the application of this technology. This work, performed at Sandia National Laboratories, was supported by the U. S. Department of Energy under contract DE-AC04-94AL85000.

\section{REFERENCES}

1. J. J. Sniegowski and E. J. Garcia, "Microfabricated Actuators and Their Application to Optics", Proc. SPIE Miniaturized Systems with Micro-Optics and Micromechanics, 2383, 2/7-9/95, San Jose, CA, (1995) pp. 46-64.

2. J. J. Sniegowski and E. J. Garcia, "Surface Micromachined Gear Trains Driven by an On-Chip Electrostatic Microengine", accepted for publication in IEEE Electron Device Letters.

3. R. Legtenberg, E. Berenschot, M.Elwenspoek and J. Fluitman, "Electrostatic Microactuators with Integrated Gear Linkages for Mechanical Power Transmission", Proc. IEEE Micro Electro Mechanical Systems (MEMS), 2/11-15/96, San Diego, CA, (1996) pp. 204-209.

4. R. L. Alley, G. J. Cuan, R. T. Howe, and K. Komvopoulos, "The Effect of Release-Etch Processing on Surface Microstructure Stiction", Technical Digest of the 1992 IEEE Solid-State Sensor and Actuator Workshop, Hilton Head Isl., SC, 6/22-25/92, (1996), pp. 202-207.

5. L. A. Field, R. M. White, and A. P. Pisano, "Fluid Powered Rotary Gears and Micro-Flow Channels", Proc. of the 6th International Conf. on Solid-State Sensors and Actuators (Transducers '91), 6/24-27/91, San Francisco, CA (1991) pp. 1033-1036.

6. E. J. Garcia and J. J. Sniegowski, "Surface Micromachined Microengine", Sensors and Actuators A, 48, 203 (1995).

7. E. J. Garcia and J. J. Sniegowski, "Surface Micromachined Microengine as the Driver for Micromechanical Gears", Proc. of the 8th International Conf. on Solid-State Sensors and Actuators and Eurosensors IX, 6/25-29/95, Stockholm, Sweden 1 (1995) pp. 365-368.

8. W. C. Tang, T-C. H. Nguyen, and R. T. Howe, "Laterally Driven Polysilicon Resonant Microstructures," Sensors and Actuators, 20, 25 (1989).

9. H. E. Merritt, Gear Engineering, Pitman Publishing, 1971, Chps. 4 and 6.

10. D. W. Dudley, Handbook of Practical Gear Design, McGraw-Hill Book Company, 1984, pp. 3.6.

11. R. D. Nasby, J. J. Sniegowski, J. H. Smith, S. Montague, C. C. Barron, W. P. Eaton, P. J. McWhorter, D. L. Hetherington, C. A. Apblett, and J. G. Fleming, "Application of Chemical-Mechanical Polishing to Planarization of Surface Micromachined Devices", Technical Digest of the 1996 SolidState Sensor and Actuator Workshop, Hilton Head Isl., SC, 6/26/96, Transducer Research Foundation (1996), pp. these Proceedings. 